KEK-TH-933

\title{
Note on Gauge Theory on Fuzzy Supersphere
}

\author{
Satoshi Iso and Hiroshi Umetsu \\ Theory Division, High Energy Accelerator Research Organization (KEK), \\ Tsukuba, Ibaraki, 305-0801, Japan \\ E-mails: satoshi.iso@kek.jp, umetsu@post.kek.jp
}

\begin{abstract}
We construct a supermatrix model whose classical background gives two-dimensional noncommutative supersphere. Quantum fluctuations around it give the supersymmetric gauge theories on the fuzzy supersphere constructed by Klimcik. This model has a parameter $\beta$ which can tune masses of the particles in the model and interpolate various supersymmetric gauge theories on sphere.
\end{abstract}




\section{Introduction}

Matrix models have been vigorously studied to understand nonperturbative aspects of string theories. In matrix models of the IKKT type [1], background space-time appears dynamically as a classical background of matrices and their fluctuations around the classical solution are regarded as gauge and matter fields on the space-time. In particular, matrix models describe noncommutative gauge theories when the classical background of matrices are noncommutative 2]. In this approach constructions of the open Wilson loop and background independence of the noncommutative gauge theories are clarified [3].

Noncommutative gauge theories appear on D-branes in string theories in a constant NS-NS two form $B$ background [4, [5] where the bosonic space-time coordinates become noncommutative. Recently it was suggested that the non anti-commutativity of the fermionic coordinates on the superspace appears in string theories in a background of the $R R$ or graviphoton field strength [6, 7, 8]. Since the non anti-commutative fermionic coordinates can be described by (super)matrices as well the noncommutative bosonic coordinates, it is expected that (super)matrix models play an important role to investigate various aspects of field theories on the noncommutative superspace. There are analyses of noncommutative superspace by using supermatrices [9-15]. Supersymmetric actions for scalar multiplets on the fuzzy two-supersphere were constructed in [9] based on the $\operatorname{ssp}(1 \mid 2)$ graded Lie algebra. Furthermore a graded differential calculus on the fuzzy supersphere is discussed in [10. Supersymmetric gauge theories on this noncommutative superspace was studied in [1] by using differential forms on it. In [12], noncommutative superspaces and their flat limits are studied by using the graded Lie algebras $o s p(1 \mid 2)$, osp $(2 \mid 2)$ and $p s u(2 \mid 2)$. Recently the concept of noncommutative superspace based on a supermatrix was also introduced in proving the Dijkgraaf-Vafa conjecture as the large N reduction [16]. Supermatrix model was also studied from the viewpoint of background independent formulations of matrix model which are expected to give constructive definitions of string theories [17].

In the previous paper [15], we constructed a supersymmetric gauge theory on a fuzzy twosupersphere based on a supermatrix model. This model has a classical solution representing a fuzzy supersphere and we obtained a supersymmetric gauge theory on the fuzzy two-supersphere expanding the supermatrices around the classical background. In a commutative limit this model is , however, different from the ordinary gauge theory in $D=2$, e.g., the action includes higher derivative terms and the fermions transform as spin $\frac{3}{2}$ representation under the $s u(2)$ isometry group on $S^{2}$. These differences are originated from the fact that we did not impose appropriate constraints on the supermatrices to eliminate extra degrees of freedom. Supermatrix formulation of supersymmetric gauge theories is similar to the covariant superspace approach for the ordinary supersymmetric gauge theories [18] because each supermatrix corresponds to the connection superfields on the superspace as we saw in our previous paper [15]. In the covariant superspace approach various constrains are imposed on the connections to eliminate redundant degrees of freedom, but in our model we could not impose appropriate conditions. After we 
wrote the previous paper [15], we were noticed the Klimcik's paper [11] where he constructed a supersymmetric gauge theory on the fuzzy supersphere by using a method of differential forms and imposing suitable constraints on the connection superfields. A crucial point of his construction is the use of the enlarged $\operatorname{osp}(2 \mid 2)$ algebra. The global $\mathcal{N}=1$ supersymmetry algebra on the fuzzy supersphere is $\operatorname{osp}(1 \mid 2)$. By adding the covariant derivatives $o s p(1 \mid 2)$ is enlarged to $\operatorname{osp}(2 \mid 2)$ because the supersymmetry generators and the covariant derivatives do not anticommute on the fuzzy supersphere. $\operatorname{Osp}(2 \mid 2)$ algebra can be regarded as $\mathcal{N}=2$ superalgebras on the sphere. Starting from the connection superfields on this $\mathcal{N}=2$ superspace, he found the correct constraints to obtain a supersymmetric gauge theory on the fuzzy supersphere.

In this paper we reformulate Klimcik's gauge theory on fuzzy supersphere in terms of supermatrix models. Very interestingly, Klimcik's gauge theory can be obtained from a supermatrix model whose classical solution gives noncommutative supersphere. Its quantum fluctuations become the supersymmetric gauge theory proposed by him. In a commutative limit, by taking a Wess-Zumino like gauge, this model becomes the ordinary supersymmetric gauge theory on $S^{2}$. In the paper we use the manifestly $S O(3)$ covariant coordinates and decompose the bosonic field $a_{i}$ into the normal component $\phi$ and tangential component $a_{i}^{(2)}$ on the sphere. The action contains a parameter which can tune masses of particles in the model.

\section{$2 \quad \operatorname{osp}(1 \mid 2)$ and $\operatorname{osp}(2 \mid 2)$ algebras}

The graded commutation relations of the $\operatorname{ssp}(2 \mid 2)$ algebra are given by

$$
\begin{array}{lll}
{\left[\hat{l}_{i}, \hat{l}_{j}\right]=i \epsilon_{i j k} \hat{l}_{k},} & {\left[\hat{l}_{i}, \hat{v}_{\alpha}\right]=\frac{1}{2}\left(\sigma_{i}\right)_{\beta \alpha} \hat{v}_{\beta},} & \left\{\hat{v}_{\alpha}, \hat{v}_{\beta}\right\}=\frac{1}{2}\left(C \sigma_{i}\right)_{\alpha \beta} \hat{l}_{i}, \\
\left\{\hat{v}_{\alpha}, \hat{d}_{\beta}\right\}=-\frac{1}{4} C_{\alpha \beta} \hat{\gamma}, & {\left[\hat{l}_{i}, \hat{d}_{\alpha}\right]=\frac{1}{2}\left(\sigma_{i}\right)_{\beta \alpha} \hat{d}_{\beta},} & \left\{\hat{d}_{\alpha}, \hat{d}_{\beta}\right\}=-\frac{1}{2}\left(C \sigma_{i}\right)_{\alpha \beta} \hat{l}_{i}, \\
{\left[\hat{\gamma}, \hat{v}_{\alpha}\right]=\hat{d}_{\alpha},} & {\left[\hat{\gamma}, \hat{d}_{\alpha}\right]=\hat{v}_{\alpha},} & {\left[\hat{\gamma}, \hat{l}_{i}\right]=0,}
\end{array}
$$

where $\hat{l}_{i}(i=1,2,3)$ and $\hat{\gamma}$ are bosonic generators, and $\hat{v}_{\alpha}$ and $\hat{d}_{\alpha}(\alpha=1,2)$ are fermionic ones. $C=i \sigma_{2}$ is the charge conjugation matrix. The $\operatorname{osp}(1 \mid 2)$ subalgebra consists of the generators $\hat{l}_{i}$ and $\hat{v}_{\alpha}$. Irreducible representations of the $\operatorname{osp}(1 \mid 2)$ algebra [19] are characterized by values of the second Casimir operator $\hat{K}_{2}=\hat{l}_{i} \hat{l}_{i}+C_{\alpha \beta} \hat{v}_{\alpha} \hat{v}_{\beta}=L\left(L+\frac{1}{2}\right)$ where quantum number $L \in \mathbb{Z}_{\geq 0} / 2$ is called super spin. Each representation of $\operatorname{osp}(1 \mid 2)$ consists of spin $L$ and $L+\frac{1}{2}$ representations of the $s u(2)$ subalgebra generated by $\hat{l}_{i}$. The dimension of the representation is $N \equiv 4 L+1$. These representations are also the so-called 'atypical representations' of $\operatorname{ssp}(2 \mid 2)$ where $\operatorname{ssp}(1 \mid 2)$ algebra can be enlarged to $\operatorname{osp}(2 \mid 2)$ algebra by adding extra generators with the same size matrices. The representation matrices of the generators $\hat{d}_{\alpha}$ and $\hat{\gamma}$ can be written as second order polynomials of the super spin $L$ representation matrices $l_{i}^{(L)}$ and $v_{\alpha}^{(L)}$ of the $\operatorname{osp}(1 \mid 2)$ generators,

$$
\gamma^{(L)}=-\frac{1}{L+1 / 4}\left(C_{\alpha \beta} v_{\alpha}^{(L)} v_{\beta}^{(L)}+2 L\left(L+\frac{1}{2}\right)\right),
$$




$$
d_{\alpha}^{(L)}=\frac{1}{2(L+1 / 4)}\left(\sigma_{i}\right)_{\beta \alpha}\left\{l_{i}^{(L)}, v_{\beta}^{(L)}\right\}
$$

The condition $\hat{K}_{2}=L(L+1 / 2)$ defines a two-dimensional supersphere. Consider polynomials $\Phi\left(l_{i}^{(L)}, v_{\alpha}^{(L)}\right)$ of the representation matrices of $l_{i}^{(L)}$ and $v_{\alpha}^{(L)}$ with super spin $L$. These polynomials are $(4 L+1) \times(4 L+1)$ supermatrices. Let us denote the space spanned by $\Phi\left(l_{i}^{(L)}, v_{\alpha}^{(L)}\right)$ as $\mathcal{A}_{L}$. The $\operatorname{osp}(1 \mid 2)$ algebra acts on $\mathcal{A}_{L}$. In particular we denote the adjoint action of the $\operatorname{osp}(1 \mid 2)$ generators as

$$
\hat{\mathcal{L}}_{i} \Phi=\left[l_{i}^{(L)}, \Phi\right], \quad \hat{\mathcal{V}}_{\alpha} \Phi=\left[v_{\alpha}^{(L)}, \Phi\right] .
$$

$\mathcal{A}_{L}$ can be decomposed into irreducible representations under the adjoint action of the $\operatorname{ssp}(1 \mid 2)$ algebra as $0 \oplus \frac{1}{2} \oplus 1 \oplus \cdots \oplus 2 L-\frac{1}{2} \oplus 2 L$. The dimension of this space is $(4 L+1)^{2}$ and thus any $(4 L+1) \times(4 L+1)$ matrices can be expanded by these polynomials. Useful basis for the expansion are the matrix super spherical harmonics $Y_{k m}^{S}\left(l_{i}^{(L)}, v_{\alpha}^{(L)}\right)$,

$$
\begin{aligned}
& \left(\hat{\mathcal{L}}_{i} \hat{\mathcal{L}}_{i}+C_{\alpha \beta} \hat{\mathcal{V}}_{\alpha} \hat{\mathcal{V}}_{\beta}\right) Y_{k m}^{S}\left(l_{i}^{(L)}, v_{\alpha}^{(L)}\right)=k\left(k+\frac{1}{2}\right) Y_{k m}^{S}\left(l_{i}^{(L)}, v_{\alpha}^{(L)}\right), \\
& \hat{\mathcal{L}}_{3} Y_{k m}^{S}\left(l_{i}^{(L)}, v_{\alpha}^{(L)}\right)=m Y_{k m}^{S}\left(l_{i}^{(L)}, v_{\alpha}^{(L)}\right) .
\end{aligned}
$$

Then $\Phi\left(l_{i}^{(L)}, v_{\alpha}^{(L)}\right)$ can be expressed as a series of the super spherical harmonics,

$$
\Phi\left(l_{i}^{(L)}, v_{\alpha}^{(L)}\right)=\sum_{k=0,1 / 2,1, \cdots}^{2 L} \phi_{k m} Y_{k m}^{S}\left(l_{i}^{(L)}, v_{\alpha}^{(L)}\right),
$$

where the Grassmann parity of the coefficient $\phi_{k m}$ is determined by the grading of the spherical harmonics. We can map the supermatrix $\Phi\left(l_{i}^{(L)}, v_{\alpha}^{(L)}\right)$ to a function on the superspace $\left(x_{i}, \theta_{\alpha}\right)$ by

$$
\Phi\left(l_{i}^{(L)}, v_{\alpha}^{(L)}\right) \longrightarrow \phi\left(x_{i}, \theta_{\alpha}\right)=\sum_{k, m} \phi_{k m} y_{k m}^{S}\left(x_{i}, \theta_{\alpha}\right),
$$

where $y_{k m}^{S}\left(x_{i}, \theta_{\alpha}\right)$ is the ordinary super spherical function. A product of supermatrices is mapped to a noncommutative star product of functions on the fuzzy supersphere [20].

In constructions of field theories on the fuzzy supersphere, the $\operatorname{ssp}(2 \mid 2)$ generators $\hat{d}_{\alpha}$ and $\hat{\gamma}$ play important roles. The adjoint action of $\hat{d}_{\alpha}$ on supermatrices is the covariant derivative on the fuzzy supersphere. The kinetic terms for a scalar multiplet on the supersphere are constructed by using these generators 9 .

\section{Supersymmetric gauge theory on fuzzy supersphere}

In this section we reformulate the Klimcik's construction of a supersymmetric gauge theory on fuzzy supersphere [11] in terms of supermatrices. We construct a supermatrix model which has a classical solution representing the fuzzy supersphere and expanding supermatrices around this background we will obtain a supersymmetric gauge theory given by Klimcik. This formulation 
has similarities to the covariant superspace approach in the ordinary supersymmetric gauge theory. Namely, we first introduce larger degrees of freedom corresponding to the connection superfields on the fuzzy supersphere. In order to eliminate redundant degrees of freedom, we need to impose appropriate constraints on them. After fixing extra gauges, we will obtain necessary degrees of freedom to describe a supersymmetric gauge theory on the noncommutative supersphere.

We first consider direct products of two vector spaces of supermatrices, $\mathcal{A}_{L}$ and $\mathcal{A}_{L^{\prime}}$. $L^{\prime}$ can be taken as any superspin. On the other hand, $L$ should be taken large in order to take a commutative limit. Each element is a $(4 L+1)\left(4 L^{\prime}+1\right) \times(4 L+1)\left(4 L^{\prime}+1\right)$ supermatrix. we then restrict to consider a special type of supermatrices which can be written as

$$
M=\sum_{A} X_{A} \otimes T^{A}
$$

where $X_{A} \in \mathcal{A}_{L}$ is a general supermatrix and $T^{A}$ is the superspin $L^{\prime}$ representation matrix of the $\operatorname{osp}(2 \mid 2)$ generators; $T^{A}=\left\{l_{i}^{\left(L^{\prime}\right)}, v_{\alpha}^{\left(L^{\prime}\right)}, d_{\alpha}^{\left(L^{\prime}\right)}, \gamma^{\left(L^{\prime}\right)}\right\}$. Among them, we can define two kinds of products, $\cdot$ and $*$,

$$
\begin{aligned}
& \left(X_{A} \otimes T^{A}\right) \cdot\left(Y_{B} \otimes T^{B}\right)=(-1)^{\left|T^{A}\right|\left|Y_{B}\right|} X_{A} Y_{B} \otimes T^{A} T^{B}, \\
& \left(X_{A} \otimes T^{A}\right) *\left(Y_{B} \otimes T^{B}\right)=(-1)^{\left|T^{A}\right|\left|Y_{B}\right|} X_{A} Y_{B} \otimes\left[T^{A}, T^{B}\right\},
\end{aligned}
$$

where $\left|T^{A}\right|$ and $\left|Y_{B}\right|$ are the gradings of $T^{A}$ and $Y_{B}$ respectively. ${ }^{1}$

The supermatrix $M$ defined above can be expanded explicitly as

$$
M=A_{i} \otimes l_{i}^{\left(L^{\prime}\right)}+C_{\alpha \beta} \varphi_{\alpha} \otimes v_{\alpha}^{\left(L^{\prime}\right)}-C_{\alpha \beta} \psi_{\alpha} \otimes d_{\beta}^{\left(L^{\prime}\right)}-\frac{1}{4} W \otimes \gamma^{\left(L^{\prime}\right)} .
$$

Here $A_{i}$ and $W$ are $(4 L+1) \times(4 L+1)$ even supermatrices, and $\varphi_{\alpha}$ and $\psi_{\alpha}$ are $(4 L+1) \times(4 L+1)$ odd supermatrices. $L^{\prime}$ is arbitrary thus one may set $L^{\prime}=\frac{1}{2}$ for simplicity. $M$ satisfies a reality condition $M^{\ddagger}=M$, that is $A_{i}^{\ddagger}=A_{i}, \varphi_{\alpha}^{\ddagger}=C_{\alpha \beta} \varphi_{\beta}, \psi_{\alpha}^{\ddagger}=C_{\alpha \beta} \psi_{\beta}$ and $W^{\ddagger}=W$. The definition of $\ddagger$ is referred to the appendix of [15]. We denote the $\operatorname{osp}(1 \mid 2)$ part of $M$ as $M_{H}$

$$
M_{H}=A_{i} \otimes l_{i}^{\left(L^{\prime}\right)}+C_{\alpha \beta} \varphi_{\alpha} \otimes v_{\alpha}^{\left(L^{\prime}\right)}
$$

and the rest as $M_{H^{\perp}} \equiv M-M_{H}$. Since all the components $A_{i}, \phi_{\alpha}, \psi_{\alpha}$ and $W$ are supermatrices and can be expanded in terms of super spin $L$ spherical harmonics, they can be regarded as superfields on the fuzzy supersphere. The supermatrix $M$ is shown to correspond to the covariant derivatives on the $\mathcal{N}=2$ extended supersphere. Then we define 'field strength' as

$$
\begin{aligned}
F & =M * M-M_{H} * M_{H}+\frac{d_{G}}{2} M-\frac{d_{H}}{2} M_{H} \\
& =\left(\frac{1}{4}\left(\sigma_{i} C\right)_{\alpha \beta}\left\{\psi_{\alpha}, \psi_{\beta}\right\}-\frac{1}{4} A_{i}\right) \otimes l_{i}^{\left(L^{\prime}\right)}+\left(-\frac{1}{4} C_{\alpha \beta}\left[\psi_{\alpha}, W\right]-\frac{1}{4} C_{\alpha \beta} \varphi_{\alpha}\right) \otimes v_{\beta}^{\left(L^{\prime}\right)}
\end{aligned}
$$

\footnotetext{
${ }^{1}$ In the paper [11], $*$ is meant for $*^{G}-*^{H}$ but in our paper we use $*$ product as $*^{G}$ or $*^{H}$ according to operated supermatrices.
} 


$$
\begin{aligned}
& +\left(\frac{1}{2}\left(\sigma_{i} C\right)_{\alpha \beta}\left[A_{i}, \psi_{\alpha}\right]+\frac{1}{4} C_{\alpha \beta}\left[\varphi_{\alpha}, W\right]-\frac{1}{2} C_{\alpha \beta} \psi_{\alpha}\right) \otimes d_{\beta}^{\left(L^{\prime}\right)} \\
& +\left(-\frac{1}{4} C_{\alpha \beta}\left\{\varphi_{\alpha}, \psi_{\beta}\right\}-\frac{1}{8} W\right) \otimes \gamma^{\left(L^{\prime}\right)}
\end{aligned}
$$

where $d_{G}=1$ and $d_{H}=\frac{3}{2}$ are Dynkin numbers.

In order to eliminate redundant superfields, we follow the prescription in [11] and impose a constraint for the $\operatorname{osp}(1 \mid 2)$ part of the field strength to vanish,

$$
\left.F\right|_{H} \equiv\left(\frac{1}{4}\left(\sigma_{i} C\right)_{\alpha \beta}\left\{\psi_{\alpha}, \psi_{\beta}\right\}-\frac{1}{4} A_{i}\right) \otimes l_{i}^{\left(L^{\prime}\right)}+\left(-\frac{1}{4} C_{\alpha \beta}\left[\psi_{\alpha}, W\right]-\frac{1}{4} C_{\alpha \beta} \varphi_{\alpha}\right) \otimes v_{\beta}^{\left(L^{\prime}\right)}=0 .
$$

This constraint breaks the $\operatorname{osp}(2 \mid 2)$ covariant structure, but still preserves the covariance under $\operatorname{osp}(1 \mid 2)$ of the model. From this constraint, $A_{i}$ and $\varphi_{\alpha}$ can be solved in terms of $\psi_{\alpha}$ and $W$ as

$$
\begin{aligned}
A_{i} & =\left(\sigma_{i} C\right)_{\alpha \beta}\left\{\psi_{\alpha}, \psi_{\beta}\right\}, \\
\varphi_{\alpha} & =-\left[\psi_{\alpha}, W\right] .
\end{aligned}
$$

Moreover we need to constrain further redundant degrees of freedom $W$ by the following condition,

$$
-\frac{1}{L^{\prime}\left(L^{\prime}+1 / 2\right)} \operatorname{STr}_{L^{\prime}}\left(M_{H^{\perp}} \cdot M_{H^{\perp}}\right)=C_{\alpha \beta} \psi_{\alpha} \psi_{\beta}+\frac{1}{4} W^{2}=L(L+1 / 2),
$$

where $\mathrm{STr}_{L^{\prime}}$ means taking a supertrace with respect to the super spin $L^{\prime}$ representation matrices. This constraint is also $\operatorname{osp}(1 \mid 2)$ invariant. In a commutative limit, this equation can be solved to eliminate $W$, as will be seen.

We now start from the following action for the supermatrix $M$;

$$
\begin{aligned}
& S=S_{F^{2}}+\beta S_{C S}, \\
& S_{F^{2}}=\operatorname{S} \operatorname{Tr}(F \cdot F), \\
& S_{C S}=\operatorname{STr}\left(\frac{2}{3} M \cdot(M * M)-\frac{2}{3} M_{H} \cdot\left(M_{H} * M_{H}\right)+\frac{d_{G}}{2} M \cdot M-\frac{d_{H}}{2} M_{H} \cdot M_{H}\right)
\end{aligned}
$$

where $\beta$ is a real constant parameter and $d_{G}$ and $d_{H}$ were defined above. This action is invariant under the following osp $(1 \mid 2)$ supersymmetry transformation,

$$
\delta M=\left[C_{\alpha \beta} \lambda_{\beta} \otimes v_{\alpha}^{\left(L^{\prime}\right)}, M\right],
$$

where a parameter $\lambda_{\alpha}$ is a $(4 L+1) \times(4 L+1)$ grading matrix multiplied by a Grassmann parameter $\tilde{\lambda}_{\alpha}$,

$$
\lambda_{\alpha}=\tilde{\lambda}_{\alpha}\left(\begin{array}{cc}
1_{2 L+1} & 0 \\
0 & -1_{2 L}
\end{array}\right) \text {. }
$$


In addition to the supersymmetry, this action has invariance under the $u(2 L+1 \mid 2 L)$ gauge transformation,

$$
\delta M=[M, H \otimes 1],
$$

where $H \in u(2 L+1 \mid 2 L)$. We note that $S_{F^{2}}$ and $S_{C S}$ are independently invariant under the supersymmetry and gauge transformations.

Then we solve the equation of motion of the above action to obtain a classical solution and expand supermatrices $M$ around it. The classical solution represents the background space-time and fluctuations are regarded as gauge fields on the space-time. One of the classical solutions is given by

$$
A_{i}=l_{i}^{(L)}, \quad \varphi_{\alpha}=v_{\alpha}^{(L)}, \quad \psi_{\alpha}=d_{\alpha}^{(L)}, \quad W=\gamma^{(L)},
$$

which represents a fuzzy supersphere. It should be noted that the solution satisfies not only the equations of motion but also the constraints (3.7) and (3.10). $M$ can be decomposed into the classical background (3.17) and fluctuation $\tilde{M}$ as

$$
\begin{aligned}
M & =M_{c l}+\tilde{M}, \\
M_{c l} & =l_{i}^{(L)} \otimes l_{i}^{\left(L^{\prime}\right)}+C_{\alpha \beta} v_{\alpha}^{(L)} \otimes v_{\beta}^{\left(L^{\prime}\right)}-C_{\alpha \beta} d_{\alpha}^{(L)} \otimes d_{\beta}^{\left(L^{\prime}\right)}-\frac{1}{4} \gamma^{(L)} \otimes \gamma^{\left(L^{\prime}\right)} .
\end{aligned}
$$

Because of the $\operatorname{ssp}(2 \mid 2)$ algebra the field strength for the classical background $M_{c l}$ vanishes,

$$
F_{c l}=M_{c l} * M_{c l}-M_{H c l} * M_{H c l}+\frac{d_{G}}{2} M_{c l}-\frac{d_{H}}{2} M_{H c l}=0,
$$

where $M_{H c l}$ is the $\operatorname{osp}(1 \mid 2)$ part of $M_{c l}$. Then the field strength (3.6) becomes

$$
\begin{aligned}
F= & \left(M_{c l} * \tilde{M}+\tilde{M} * M_{c l}+\frac{d_{G}}{2} \tilde{M}\right)-\left(M_{H c l} * \tilde{M}_{H}+\tilde{M}_{H} * M_{H c l}+\frac{d_{H}}{2} \tilde{M}_{H}\right) \\
& +\tilde{M} * \tilde{M}-\tilde{M}_{H} * \tilde{M}_{H},
\end{aligned}
$$

where $\tilde{M}_{H}$ is the $\operatorname{osp}(1 \mid 2)$ part of $\tilde{M}$. In this form, terms in the first and second parenthesises respectively coincide with $\delta^{G} \tilde{M}$ and $\delta^{H} \tilde{M}_{H}$ in Klimcik's notation [11] and thus this is nothing but the field strength defined by him.

Expanding each component of the supermatrices around the classical background,

$$
A_{i}=l_{i}^{(L)}+\tilde{A}_{i}, \quad \varphi_{\alpha}=v_{\alpha}^{(L)}+\tilde{\varphi}_{\alpha}, \quad \psi_{\alpha}=d_{\alpha}^{(L)}+\tilde{\psi}_{\alpha}, \quad W=\gamma^{(L)}+\tilde{W},
$$

$\left.F\right|_{H^{\perp}}$ and $S_{C S}$ become

$$
\begin{aligned}
\left.F\right|_{H^{\perp}}= & {\left[\frac{1}{2}\left(\sigma_{i} C\right)_{\alpha \beta}\left(\left[l_{i}^{(L)}, \tilde{\psi}_{\alpha}\right]-\left[d_{\alpha}^{(L)}, \tilde{A}_{i}\right]\right)+\frac{1}{4} C_{\alpha \beta}\left(\left[v_{\alpha}^{(L)}, \tilde{W}\right]-\left[\gamma^{(L)}, \tilde{\varphi}_{\alpha}\right]\right)-\frac{1}{2} C_{\alpha \beta} \tilde{\psi}_{\alpha}\right] \otimes d_{\beta}^{\left(L^{\prime}\right)} } \\
& +\left[-\frac{1}{4} C_{\alpha \beta}\left(\left\{v_{\alpha}^{(L)}, \tilde{\psi}_{\beta}\right\}+\left\{d_{\beta}^{(L)}, \tilde{\varphi}_{\beta}\right\}\right)-\frac{1}{8} \tilde{W}\right] \otimes \gamma^{\left(L^{\prime}\right)}, \\
S_{C S}= & \frac{1}{4} L^{\prime}\left(L^{\prime}+1 / 2\right) \operatorname{STr}_{L}\left(2\left(\sigma_{i} C\right)_{\alpha \beta} \tilde{A}_{i}\left\{\tilde{\psi}_{\alpha}, \tilde{\psi}_{\beta}\right\}-2 C_{\alpha \beta} \tilde{W}\left\{\tilde{\varphi}_{\alpha}, \tilde{\psi}_{\beta}\right\}\right. \\
& -2\left(\sigma_{i} C\right)_{\alpha \beta} \tilde{\psi}_{\alpha}\left[l_{i}^{(L)}, \tilde{\psi}_{\beta}\right]+4\left(\sigma_{i} C\right)_{\alpha \beta} \tilde{A}_{i}\left\{d_{\alpha}^{(L)}, \tilde{\psi}_{\beta}\right\} \\
& +2 C_{\alpha \beta} \tilde{\varphi}_{\alpha}\left[\gamma^{(L)}, \tilde{\psi}_{\beta}\right]-2 C_{\alpha \beta} \tilde{W}\left\{v_{\alpha}^{(L)}, \tilde{\psi}_{\beta}\right\}-2 C_{\alpha \beta} \tilde{W}\left\{d_{\beta}^{(L)}, \tilde{\varphi}_{\alpha}\right\} \\
& \left.-\tilde{A}_{i} \tilde{A}_{i}-C_{\alpha \beta} \tilde{\varphi}_{\alpha} \tilde{\varphi}_{\beta}-2 C_{\alpha \beta} \tilde{\psi}_{\alpha} \tilde{\psi}_{\beta}-\frac{1}{2} \tilde{W}^{2}\right) .
\end{aligned}
$$


Note that the supermatrices $A_{i}, \phi_{\alpha}, \psi_{\alpha}$ and $W$ are covariant derivatives on $\mathcal{N}=2$ superspace since they transform covariantly under the $U(2 L+1 \mid 2 L)$ gauge transformations.

Because of the constraints (3.7) and (3.10), $\tilde{\psi}_{\alpha}$ is the only independent supermatrix and the others can be solved in terms of $\tilde{\psi}_{\alpha}$;

$$
\begin{aligned}
& \tilde{A}_{i}=\left(\sigma_{i} C\right)_{\alpha \beta}\left(2\left\{d_{\alpha}^{(L)}, \tilde{\psi}_{\beta}\right\}+\left\{\tilde{\psi}_{\alpha}, \tilde{\psi}_{\beta}\right\}\right), \\
& \tilde{\varphi}_{\alpha}=-\left[d_{\alpha}^{(L)}, \tilde{W}\right]+\left[\gamma^{(L)}, \tilde{\psi}_{\alpha}\right]-\left[\tilde{\psi}_{\alpha}, \tilde{W}\right], \\
& C_{\alpha \beta}\left[d_{\alpha}^{(L)}, \tilde{\psi}_{\beta}\right]+\frac{1}{4}\left\{\gamma^{(L)}, \tilde{W}\right\}+C_{\alpha \beta} \tilde{\psi}_{\alpha} \tilde{\psi}_{\beta}+\frac{1}{4} \tilde{W}^{2}=0 .
\end{aligned}
$$

$\tilde{\psi}_{\alpha}$ is an odd supermatrix which can be expanded by $l_{i}^{(L)}$ and $v_{\alpha}^{(L)}$, thus it is regarded as a spinor superfield on the fuzzy supersphere. The supermatrix $\tilde{W}$ can not be explicitly solved in general because of the quadratic term but we will show that it can be solved in a commutative limit. For a while we treat both of $\tilde{\psi}_{\alpha}$ and $\tilde{W}$ as independent variables.

Though fixing the classical background as above violates the original osp (1|2) supersymmetry (3.14), it can be compensated by appropriate $u(2 L+1 \mid 2 L)$ gauge transformations. Actually the action is invariant under the following combined transformations,

$$
\begin{aligned}
& \delta \tilde{\psi}_{\alpha}=\frac{1}{4} \lambda_{\alpha} \tilde{W}+C_{\beta \gamma} \lambda_{\gamma}\left\{v_{\beta}^{(L)}, \tilde{\psi}_{\alpha}\right\}, \\
& \delta \tilde{W}=C_{\alpha \beta} \lambda_{\beta} \tilde{\psi}_{\alpha}+C_{\alpha \beta} \lambda_{\beta}\left[v_{\alpha}^{(L)}, \tilde{W}\right] .
\end{aligned}
$$

The action is also invariant under gauge transformations,

$$
\delta \tilde{\psi}_{\alpha}=\left[d_{\alpha}^{(L)}+\tilde{\psi}_{\alpha}, H\right], \quad \delta \tilde{W}=\left[\gamma^{(L)}+\tilde{W}, H\right],
$$

for $H \in u(2 L+1 \mid 2 L)$. These transformations are compatible with the constraints. The fact that the model has the $\operatorname{osp}(1 \mid 2)$ supersymmetry even after choosing the classical background can be also understood as the following. The elements with the form $u_{i} l_{i}^{(L)}+C_{\alpha \beta} \lambda_{\alpha} v_{\beta}^{(L)}$ in $u(2 L+1 \mid 2 L)$ constitute the $\operatorname{osp}(1 \mid 2)$ subalgebra. Thus the model originally has two independent $o s p(1 \mid 2)$ symmetries. The classical background preserves a half of these symmetries which is given by the transformations (3.28).

Mapping from the supermatrix model to a noncommutative field theory on the supersphere is performed by the same method as ones used in [15. The classical solutions $l_{i}^{(L)}$ and $v_{\alpha}^{(L)}$ are mapped to coordinates $\left(x_{i}, \theta_{\alpha}\right)$ on the supersphere,

$$
\begin{aligned}
x_{i} & =\frac{1}{\sqrt{L(L+1 / 2)}} l_{i}^{(L)}, \\
\theta_{\alpha} & =\frac{1}{\sqrt{L(L+1 / 2)}} v_{\alpha}^{(L)},
\end{aligned}
$$

where we set a radius of the supersphere to 1 for simplicity, that is $x_{i} x_{i}+C_{\alpha \beta} \theta_{\alpha} \theta_{\beta}=1$. And $d_{\alpha}^{(L)}$ and $\gamma^{(L)}$ are written as second order polynomials of $x_{i}$ and $\theta_{\alpha}$ according to (2.2) and (2.3). 
The adjoint actions of these $\operatorname{ssp}(2 \mid 2)$ generators are mapped to the differential operators on the supersphere. The supertrace becomes an integration on the supersphere,

$$
\mathrm{STr}_{L} \longrightarrow-\frac{1}{2 \pi} \int d^{3} x d^{2} \theta \delta\left(x^{2}+\theta^{2}-1\right)
$$

where $\theta^{2}=C_{\alpha \beta} \theta_{\alpha} \theta_{\beta}$. The supermatrix $\tilde{\psi}_{\alpha}$ is mapped to a spinor superfield which can be regarded as the spinor connection on the fuzzy supersphere. The field theory action derived by these procedure is written in terms of this spinor superfield. The construction of the supersymmetric gauge theory on the fuzzy supersphere given here is a natural extension of the covariant superspace approach for the ordinary super Yang-Mills theory in a flat space-time.

Next we consider a commutative limit to see that our model is indeed noncommutative generalization of the ordinary supersymmetric gauge theory on sphere. A commutative limit is given by taking $L \rightarrow \infty$ limit keeping the radius of the supersphere fixed. In this limit superfields $\tilde{\psi}_{\alpha}$ and $\tilde{W}$ are expanded as

$$
\begin{aligned}
& \tilde{\psi}_{\alpha}(x, \theta)=\eta_{\alpha}(x)+\left(\sigma_{\mu}\right)_{\beta \alpha} a_{\mu}(x) \theta_{\beta}+\left(\xi_{\alpha}(x)+\frac{1}{2 r^{2}} x_{i} \partial_{i} \eta_{\alpha}(x)\right) \theta^{2}, \\
& \tilde{W}(x, \theta)=w(x)+C_{\alpha \beta} \zeta_{\alpha}(x) \theta_{\beta}+\left(F(x)+\frac{1}{2 r^{2}} x_{i} \partial_{i} w(x)\right) \theta^{2},
\end{aligned}
$$

where $r^{2}=x_{i} x_{i}$ and $\mu=0,1,2,3 . a_{\mu}, w, F$ are bosonic and $\eta_{\alpha}, \xi_{\alpha}, \zeta_{\alpha}$ are fermionic fields. The $u(2 L+1 \mid 2 L)$ gauge parameter is also expanded as

$$
H(x, \theta)=h(x)+C_{\alpha \beta} h_{\alpha}(x) \theta_{\beta}+f(x) \theta^{2} .
$$

The gauge transformation generated by $h(x)$ is an ordinary gauge transformation while the others are supersymmetric extension of it. To fix these extra gauge degrees of freedom generated by $h_{\alpha}$ and $f$, we set the Wess-Zumino like gauge fixing condition $C_{\alpha \beta} \theta_{\alpha} \tilde{\psi}_{\beta}=0$, thus $\eta_{\alpha}=a_{0}=0$. In the commutative limit the constraint (3.27) can be solved as $\tilde{W}=2 x_{i}\left(\sigma_{i} C\right)_{\alpha \beta} \theta_{\alpha} \tilde{\psi}_{\beta}$, so that

$$
w=\zeta_{\alpha}=0, \quad F=-2(x \cdot a) \theta^{2} .
$$

Here we used the fact that the fields $\tilde{\psi}_{\alpha}$ and $\tilde{W}$ scale as $\mathcal{O}(1)$ while $d_{\alpha}^{(L)}$ and $\gamma^{(L)}$ scale as $\mathcal{O}(L)$ in $L \rightarrow \infty$. By the gauge fixing condition and the constraint, the independent fields are now $a_{i}(x)$ and $\xi_{\alpha}(x)$.

We then decompose $a_{i}$ into a scalar field $\phi$ and a gauge field $a_{i}^{(2)}$ on $S^{2}$,

$$
a_{i}=n_{i} \phi+a_{i}^{(2)}
$$

where $n_{i}$ is a unit normal vector on the sphere and $n_{i} a_{i}^{(2)}=0$ and $a_{i}^{(2)}$ is a tangential projection; $a_{i}^{(2)}=\left(\delta_{i j}-n_{i} n_{j}\right) a_{j}$. The $U(1)$ field strength for the tangential component is defined as $F_{i j}^{(2)}=$ $R_{i} a_{j}^{(2)}-R_{j} a_{i}^{(2)}-i \epsilon_{i j k} a_{k}^{(2)}$ where $R_{i}=-i \epsilon_{i j k} n_{i} \frac{\partial}{\partial n_{i}}$ are derivatives on $S^{2}$. After straightforward 
calculations, we obtain the action in the commutative limit,

$$
\begin{aligned}
S= & \frac{L^{\prime}\left(L^{\prime}+1 / 2\right)}{4 \pi} \int d \Omega\left\{\frac{9}{8} F_{i j}^{(2)} F_{i j}^{(2)}-\frac{i}{4}\left(\epsilon_{i j k} n_{i} F_{j k}^{(2)}\right) \phi+\frac{9}{4}\left(R_{i} \phi\right)^{2}-\frac{1}{4} \phi^{2}\right. \\
& -\frac{9}{4}\left(\sigma_{i} C\right)_{\alpha \beta} \xi_{\alpha} R_{i} \xi_{\beta}-\frac{1}{4} C_{\alpha \beta} \xi_{\alpha} \xi_{\beta} \\
& \left.-3 \beta\left[i\left(\epsilon_{i j k} n_{i} F_{j k}^{(2)}\right) \phi+\phi^{2}+C_{\alpha \beta} \xi_{\alpha} \xi_{\beta},\right]\right\} .
\end{aligned}
$$

This model consists of a $U(1)$ gauge field which has no local degrees of freedom, a scalar and a Majorana fermion. There is a free tunable parameter $\beta$. Mass of the fermion is given by $\sqrt{\mu(\mu-1)}$ with $\mu=(1+12 \beta) / 9$ because of

$$
\left\langle\xi_{\alpha}(x) \xi_{\beta}(y)\right\rangle \sim \frac{\left(C \sigma_{i}\right)_{\alpha \beta} R_{i}+(1-\mu) C_{\alpha \beta}}{R_{i} R_{i}-\mu(\mu-1)} .
$$

The massless Dirac operator is given by $D=\sigma_{i} R_{i}+1$. The second term in $D$ comes from the spin connection on the sphere and this $D$ anticommutes with the chirality operator $\sigma_{i} n_{i}$. By integrating out the scalar field $\phi$ it can be shown that the bosonic supersymmetric parter of $\xi$ has the same mass. For $\beta=2 / 3$ the action becomes the one given in [21]. On the other hand, for $\beta=-1 / 12$ the mixing term between $\phi$ and $a_{i}^{(2)}$ disappears. For both cases, bosonic and fermionic excitations are massless.

Since the gauge fixing condition we chose is not invariant under the supersymmetry transformations (3.28), we must compensate them by the field-dependent gauge transformation with the following gauge parameter,

$$
\begin{aligned}
& h_{\alpha}=-\lambda_{\alpha} \phi-i \epsilon_{i j k}\left(\sigma_{k}\right)_{\beta \alpha} \lambda_{\beta} n_{i} a_{j}^{(2)}, \\
& f=\frac{1}{2}\left(\sigma_{i} C\right)_{\alpha \beta} \lambda_{\beta} n_{i} \xi_{\alpha} .
\end{aligned}
$$

Then the supersymmetry transformations become

$$
\begin{aligned}
\delta_{\lambda} \phi & =-\frac{1}{2}\left(C_{\alpha \beta} \lambda_{\beta} \xi_{\alpha}\right) \\
\delta_{\lambda} a_{i}^{(2)} & =-\frac{i}{2} \epsilon_{i j k} n_{j}\left(\sigma_{k} C\right)_{\alpha \beta} \lambda_{\beta} \xi_{\alpha}, \\
\delta_{\lambda} \xi_{\alpha} & =-\frac{1}{2} \lambda_{\alpha}\left(\phi+\frac{i}{2} \epsilon_{i j k} n_{i} F_{j k}^{(2)}\right)+\frac{1}{2}\left(\sigma_{i}\right)_{\beta \alpha} \lambda_{\beta} R_{i} \phi .
\end{aligned}
$$

The $U(1)$ gauge transformation is given by

$$
\delta a_{i}^{(2)}=R_{i} h, \quad \delta \phi=\delta \xi_{\alpha}=0 .
$$

The action (3.39) is invariant under these transformations. It can be shown that the commutation relations between two supersymmetry transformations generate the translations on $S^{2}$ up to gauge transformations,

$$
\begin{aligned}
{\left[\delta_{\lambda^{1}}, \delta_{\lambda^{2}}\right] \phi } & =\Theta_{i} R_{i} \phi \\
{\left[\delta_{\lambda^{1}}, \delta_{\lambda^{2}}\right] a_{i}^{(2)} } & =\Theta_{j} R_{j} a_{i}^{(2)}+i \epsilon_{i j k} \Theta_{j} a_{k}^{(2)}+R_{i}\left[\Theta_{j}\left(n_{j} \phi-a_{j}^{(2)}\right)\right] \\
{\left[\delta_{\lambda^{1}}, \delta_{\lambda^{2}}\right] \xi_{\alpha} } & =\Theta_{i} R_{i} \xi_{\alpha}+\frac{1}{2} \Theta_{i}\left(\sigma_{i}\right)_{\beta \alpha} \xi_{\beta},
\end{aligned}
$$


with $\Theta_{i}=-\frac{1}{2}\left(\sigma_{i} C\right)_{\alpha \beta} \lambda_{\alpha}^{2} \lambda_{\beta}^{1}$. We again note that the action has these symmetries independently of the parameter $\beta$.

In this paper we concentrated on the case of $U(1)$ gauge theory, but it is easily generalized to supersymmetric gauge theory with $U(k)(k>1)$ group.

\section{Summary and discussions}

In this paper, we constructed a supersymmetric gauge theory on fuzzy supersphere from a supermatrix model. Our construction of the supermatrix model is based on the prescription given by Klimcik [1]. We have shown that the fuzzy supersphere can be obtained as a classical solution of the supermatrix model and fluctuations around it become supersymmetric gauge fields on the supersphere. Namely, both of the Klimcik's gauge theory and fuzzy supersphere background are shown to be derivable from a single supermatrix. In this sense, this model has background independence as well as the other known gauge theories on noncommutative space-time.

The formulation adopted here is similar to the so called covariant superspace approach to supersymmetric gauge theories on superspace. Namely we first introduce larger degrees of freedom corresponding connection superfields on the superspace and then impose various constraints on the superfields. In our case, we follow the prescription by Klimcik and start from connection superfields on $\mathcal{N}=2$ superspace on the sphere to obtain $\mathcal{N}=1$ supersymmetric field theory. In this way, we can impose appropriate constraints to eliminate redundant fields. In our previous paper [15], we started from less number of superfields corresponding to connections on $\mathcal{N}=1$ but could not impose the appropriate constraint compatible with $\operatorname{osp}(1 \mid 2)$ global supersymmetry. This is in contrast with the ordinary flat case where we can impose appropriate constraints on the connection superfields on $\mathcal{N}=1$ superspace.

Our construction may be generalizable to higher dimensional curved cases. For example, it will be interesting to consider a supermatrix model based on $p s u(2 \mid 2)$ super Lie algebra to obtain a supersymmetric gauge theory on noncommutative superspace of $A d S^{2} \times S^{2}$.

\section{Acknowledgments}

We would like to thank C. Klimcik for informing us of his paper [11. The work of H.U. is supported in part by JSPS Research Fellowships for Young Scientists.

\section{References}

[1] N. Ishibashi, H. Kawai, Y. Kitazawa and A. Tsuchiya, Nucl. Phys. B 498, 467 (1997) arXiv:hep-th/9612115. 
[2] H. Aoki, N. Ishibashi, S. Iso, H. Kawai, Y. Kitazawa and T. Tada, Nucl. Phys. B 565, 176 (2000) arXiv:hep-th/9908141.

[3] N. Ishibashi, S. Iso, H. Kawai and Y. Kitazawa, Nucl. Phys. B 573, 573 (2000) arXiv:hep-th/9910004.

[4] V. Schomerus, JHEP 9906 (1999) 030 arXiv:hep-th/9903205.

[5] N. Seiberg and E. Witten, JHEP 9909 (1999) 032 arXiv:hep-th/9908142.

[6] H. Ooguri and C. Vafa, Adv. Theor. Math. Phys. 7, 53 (2003) arXiv:hep-th/0302109. H. Ooguri and C. Vafa, arXiv:hep-th/0303063.

[7] J. de Boer, P. A. Grassi and P. van Nieuwenhuizen, Phys. Lett. B 574, 98 (2003) arXiv:hep-th/0302078.

[8] N. Seiberg, JHEP 0306, 010 (2003) arXiv:hep-th/0305248.

[9] H. Grosse, C. Klimcik and P. Presnajder, Commun. Math. Phys. 185, 155 (1997) arXiv:hep-th/9507074.

[10] H. Grosse and G. Reiter, Jour. Geom. Phys. 28, 349 (1998)

[11] C. Klimcik, Commun. Math. Phys. 206567 (1999) arXiv:hep-th/9903112.

K. Valavane, Class. Quant. Grav. 17, 4491 (2000) arXiv:hep-th/0006245.

[12] M. Hatsuda, S. Iso and H. Umetsu, Nucl. Phys. B 671217 (2003) arXiv:hep-th/0306251.

[13] J. H. Park, JHEP 0309 (2003) 046 arXiv:hep-th/0307060.

[14] Y. Shibusa and T. Tada, Phys. Lett. B 579, 211 (2004) arXiv:hep-th/0307236.

[15] S. Iso and H. Umetsu, arXiv:hep-th/0311005.

[16] H. Kawai, T. Kuroki and T. Morita, Nucl. Phys. B 664185 (2003) arXiv:hep-th/0303210.

H. Kawai, T. Kuroki and T. Morita, arXiv:hep-th/0312026.

[17] L. Smolin, arXiv:hep-th/0006137. T. Azuma, S. Iso, H. Kawai and Y. Ohwashi, Nucl. Phys. B 610, 251 (2001) arXiv:hep-th/0102168.

[18] For example, S. J. Gates, M. T. Grisaru, M. Rocek and W. Siegel, "Superspace, Or One Thousand And One Lessons In Supersymmetry," Front. Phys. 58 (1983) 1 arXiv:hep-th/0108200.

M. F. Sohnius, Phys. Rept. 128 (1985) 39.

J. Wess and J. Bagger, "Supersymmetry And Supergravity,"

[19] A. Pais and V. Rittenberg, J. Math. Phys. 16, 2062 (1975) [Erratum-ibid. 17, 598 (1976)]. M. Scheunert, W. Nahm and V. Rittenberg, J. Math. Phys. 18, 155 (1977). M. Marcu, J. Math. Phys. 21, 1277 (1980). 
[20] A. P. Balachandran, S. Kurkcuoglu and E. Rojas, JHEP 0207, 056 (2002) arXiv:hep-th/0204170.

[21] S. Iso, Y. Kimura, K. Tanaka and K. Wakatsuki, Nucl. Phys. B 604, 121 (2001) arXiv:hep-th/0101102. 